\title{
PREDICTION OF STAND PIPE PRESSURE USING CONVENTIONAL APPROACH
}

\author{
Dipankar Chowdhury, ${ }^{* 1}$, Pål Skalle ${ }^{1}$, Mohammed Mahbubur Rahman² \\ ${ }^{1}$ Department of Petroleum Engineering and Applied Geophysics, Norwegian University of Science and Technology \\ Trondheim-7491, Norway \\ ${ }^{2}$ Department of Petroleum and Mineral Resources Engineering, Bangladesh University of Engineering and Technology \\ Dhaka-1000, Bangladesh
}

Received 21 June 2009; received in revised form 19 July 2009

\begin{abstract}
In rotary drilling operation, the hydraulic circuit typically consists of stand pipe, rotary hose, swivel, Kelly, drill pipe, drill collar, drill bit, and the annulus between the drillstring and the open hole or the casing. Stand Pipe Pressure, abbreviated as SPP, is defined as the total frictional pressure drop in the hydraulic circuit. SPP, an important drilling parameter in selecting proper mud weight, can be calculated using different rheological models. In this paper, the results obtained using the four widely used rheological models namely the Newtonian model, the Bingham plastic model, the Power law model and the Herschel-Bulkley model are presented. The rheological data used are collected by performing circulation test while drilling a vertical well in the Po valley, Italy. The rheological constants associated with each of the four models are calculated using regression analysis, For the three flow rates used during the circulation test, SPP has been predicted with a maximum error of $1.2 \%$ when compared with the measured values. The Bingham plastic model produces best SPP estimates for all the three flow rates for the drilling condition considered.
\end{abstract}

Keywords: SPP, rheological models, frictional pressure drop

DOI:10.3329/cerb.v13i1.2703

\section{Introduction}

When drilling fluid circulates, pressure drop takes place due to friction between the fluid and the surface in contact. The pressure that forces the drilling fluid to circulate through the hydraulic system is supplied by the mud pump. The mud pump pressure is partly used up in overcoming the friction between the fluid, and the open hole or casing or the surface equipment used. The remaining pump pressure is consumed as bit nozzle pressure loss, where the high nozzle speed is assisting in cuttings removal from the bit and its surroundings. The total pressure drop that occurs due to fluid friction is termed as Stand Pipe Pressure or SPP.

SPP is an important drilling parameter that must be known with sufficient accuracy for selecting proper jet bit nozzle size, determining optimum flow rate to ensure efficient hole cleaning and selecting proper mud pump liner. Continuous monitoring of SPP also helps in identifying downhole problems. For example, too low SPP can be caused by washed out pipe or bit nozzle, loose joint or broken drill string, worn pump packing or liner, and lost returns due to formation fracture. On the other hand, too high SPP could indicate a plugged drill bit or an increase in mud density or viscosity. Reliable indication of SPP provides an early

${ }^{*}$ Corresponding author Email: dipu049@yahoo.com warning of circulation problems and thus warns the driller to make corrections avoiding major problems.

Rheology, the science of flow and deformation of matter, deals with stress-strain relationships of drilling fluid. Different rheological models are used for characterization of a drilling fluid. Proper determination of rheological constants is necessary for calculating SPP.

Okafor and Evers [1] performed an experimental comparison among three rheological models: i) Robertson-Stiff, ii) Power law and iii) Bingham Plastic model. They used two types of clay-water drilling fluids. They found that the most accurate frictional pressure loss was predicted by Robertson-Stiff model. They also observed that Robertson-Stiff and Power Law model predicted frictional pressure loss with no significant difference at typical oil well circulating velocities of 0.061 to $0.24 \mathrm{~m} / \mathrm{s}$.

Maglione and Robotti [2] developed a methodology to determine the three constants of the HerschelBulkley model using the data from the circulation test conducted for three flow rates. They calculated SPP for a drilling well located at the Po valley in Italy using the same model.

In this paper, the results obtained for four rheological models are presented. The four models used are: i) Newtonian model, ii) Bingham plastic model, iii) Power law model and iv) Herschel-Bulkley model. The models are tested to find out their ability in providing the best SPP estimate for the water based (C)Bangladesh Uni. of Engg. $\mathcal{G}$ Tech. 
drilling fluid, drillstring, and the surface equipment geometry used to drill a vertical well in the Po valley of Italy [2].

Even though it is well known that drilling muds do not behave as Newtonian fluids, the Newtonian model is chosen to investigate about the theoretical pressure drop if a Newtonian fluid were used. The use of Bingham Plastic model and Power law model is a common practice for most oil companies. However, these models tend to represent the drilling fluid behavior inaccurately especially at low and medium shear rate ranges [3]. The Herschel-Bulkley model is chosen as it incorporates the nonlinear shear stress-shear rate relationship and the yield shear stress exhibited by most drilling fluids.

\section{The Rheological Models}

A rheological model describes the relationship between shear stress and shear rate when a fluid flows through a circular section or an annulus. Among the various rheological models, this paper considers the four most widely considered rheological models.

\subsection{Newtonian model}

It describes a fluid considering a linear relationship between shear stress $(\tau)$ and shear rate $(\gamma)$. Graphically this is represented by a straight line passing through the origin with a slope equal to the dynamic viscosity $(\mu)$ of the fluid. This can be expressed as

$$
\tau=\mu \gamma
$$

This linear relation is valid only as long as the fluid flow is laminar [4]. Laminar flow occurs at low shear rates. At high shear rates, the flow pattern changes from laminar to turbulent. For a Newtonian fluid, viscosity is constant and is only influenced by changes in temperature and pressure [5].

\subsection{Bingham plastic model}

It is used to approximate the pseudoplastic behavior (i.e. decrease of apparent viscosity with increasing shear rate) of drilling fluids and cement slurries [4]. The Bingham plastic model is defined as

$$
\tau=\mu_{\mathrm{p}} \gamma+\tau_{\mathrm{y}}
$$

The above mathematical expression is valid only for laminar flow [4]. The Bingham plastic fluid requires the applied shear stress to exceed a certain minimum value so that the fluid can flow. This minimum value is called the yield point $\left(\tau_{\mathrm{y}}\right)$. Once the yield point is exceeded, changes in shear stress are proportional to changes in shear rate. The constant of proportionality is called the plastic viscosity $\left(\mu_{\mathrm{p}}\right)$. The plastic viscosity depends on pressure and temperature. The Bingham plastic model works well for higher shear rates but gives a significant error at low shear rates. It also may predict a non-physical yield point [6].

\subsection{Power law model}

It is used to approximate the pseudoplastic behavior of drilling fluids and cement slurries [4]. This model is defined as

$$
\tau=K \gamma^{m}
$$

This model uses two parameters for fluid characterization- the consistency index $(K)$ and the flow behavior index $(m)$. When $m=1$, the above equation reduces to the Newtonian model. Besides, the Power law model can be used to represent a pseudoplastic fluid $(m<1)$ and also a dilatant fluid $(m>1)$.

$K$ describes the thickness of the fluid and is analogous to apparent viscosity of the fluid. Large values of $K$ mean that the fluid is very thick. The value of $m$ indicates the degree of Non-Newtonian behavior of the fluid. The shortcoming of Power law model is that it underestimates the shear stresses at medium and low shear rate ranges [3]. The Power law model is also known as the Ostwald-de Walle model.

\subsection{Herschel-Bulkley model}

It is also known as the yield Power law model, as it is a hybrid of the Bingham Plastic and the Power law models [7]. The Power law model does not consider the yield point while the Herschel-Bulkley model takes the yield point into account. This model is defined as

$$
\tau=\tau_{\mathrm{y}}+K \gamma^{m}
$$

The Herschel-Bulkley model is reduced to the Power law model when $\tau_{\mathrm{y}}=0$ and to the Bingham plastic model when $m=1$. It is to be noted that this model can yield mathematical expressions that are not readily solved analytically but can be solved using non-linear regression [7].

A graphical representation of the four rheological models is presented in Figure 1.

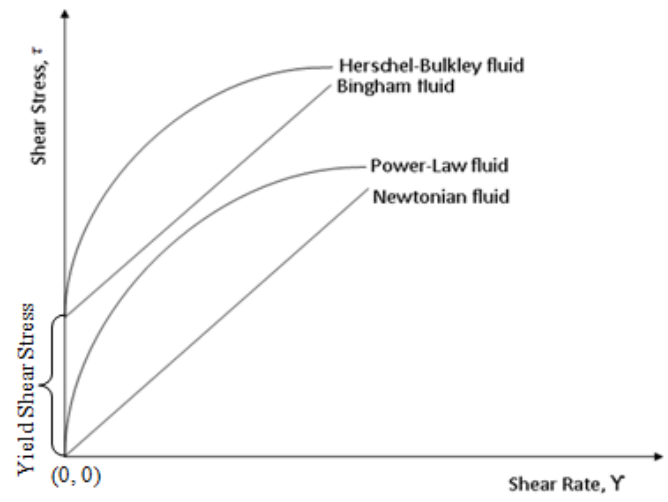

Figure 1: Graphical comparison of the four rheological models 


\section{Field Data and Operational Conditions}

SPP has been measured for three different flow rates while performing a circulation test in the $17^{1} / 2^{\text {" sec- }}$ tion of the well at a depth of $798 \mathrm{~m}$. During the test, the following conditions are maintained:

- The drilling fluid is circulated with the bit off bottom while making a run in hole trip.

- During the test, the drill string is not rotated.

- The mud logging unit monitored the stand pipe pressure readings.

- The mud sample used for rheological measurement is taken from the shale shaker outlet during the circulation test.

Figure 2 shows the geometrical dimensions of the cased and open hole sections. The casing and hole dimensions, the mud rheology measurements, the drillstring dimensions, and the measured SPPs alongwith the corresponding flow rates are presented in Tables 1 , 2, 3 and 4 respectively in the Appendix and adopted from Maglione and Robotti [2].

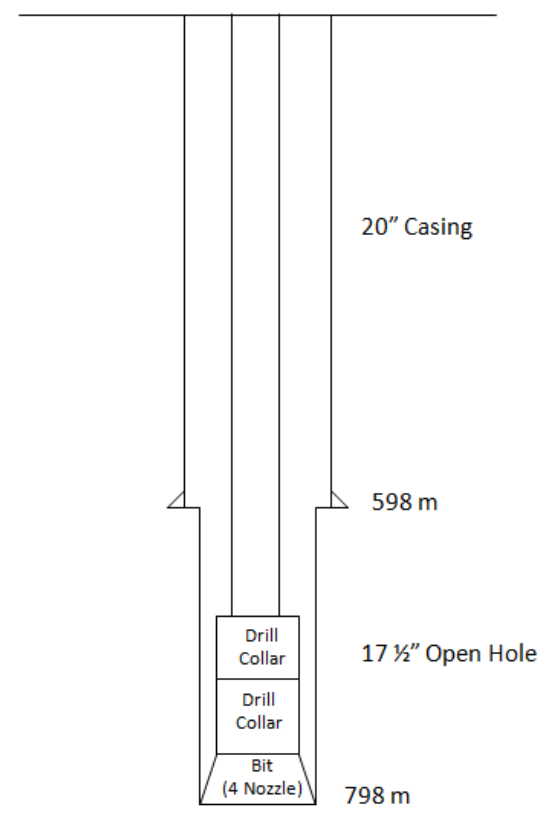

Figure 2: Casing and hole dimensions

\section{SPP Prediction}

The following steps are used for predicting SPP for the three flow rates used during the circulation test:

\subsection{Determination of the rheological constants}

The initial estimates of the rheological constants are determined using the field approach. In this approach, the Fann viscometer readings for 600 and $300 \mathrm{rpm}$ are used. These intial estimates are then used for getting the final values of the rheological constants using regression analysis. The regression analysis is performed over all the six readings of the Fann viscometer. For regression analysis, MS Excel has been used. A detail of the approach used can be found in literature [8].

\subsection{Prediction of SPP}

A Fortran 95 code is developed to predict SPP for the drilling condition under consideration. SPP is predicted using the frictional pressure drop relations developed for the four rheological models considered. This phenomenon is well explained in the literature [9-13].

\section{Results and Discussion}

The rheological constants obtained and the predicted SPP by the four models are presented in Table 5 and 6 respectively in the Appendix. It can be seen that the Newtonian model produces the worst estimate for each of the three flow rates. It is quite logical, since drilling muds are Non-Newtonian fluids. Among the other three models, the Bingham plastic model produces the best estimate for each flow rate with a maximum absolute error of less than one bar. For the Power law model, the highest absolute error found is approximately 16 bars at 3270 liter/min while it is approximately 13 bars for the Herschel-Bulkley model at the same flow rate. The predictions made by the Power law model and the Herschel-Bulkley model are considerably close to each other with a maximum discrepancy of 2.9 bars. The absolute error in predicted SPP by the Bingham plastic model, the Power law model and the Herschel-Bulkley model is shown in Figure 3. The figure shows the large amount of error in predicted SPP by the Power law model and the Herschel-Bulkley model at the two higher flow rates.

One interesting observation is made. As indicated by Figure 4, the best rheogram is produced by the Herschel-Bulkley model with an $\mathrm{R}^{2}$ value of 0.999 . However, the Bingham model with an $\mathrm{R}^{2}$ value of 0.945 produces the best SPP estimates for all the flow rates considered. The $\mathrm{R}^{2}$ value for the Power law model is 0.996. In this figure, 'Fann' indicates the actual rheological measurements obtained by the Fann viscometer.

There are two major limitations of this work. First, the results obtained are limited to the single water based mud used during the circulation test and the drilling scenario considered. Second, the frictional 


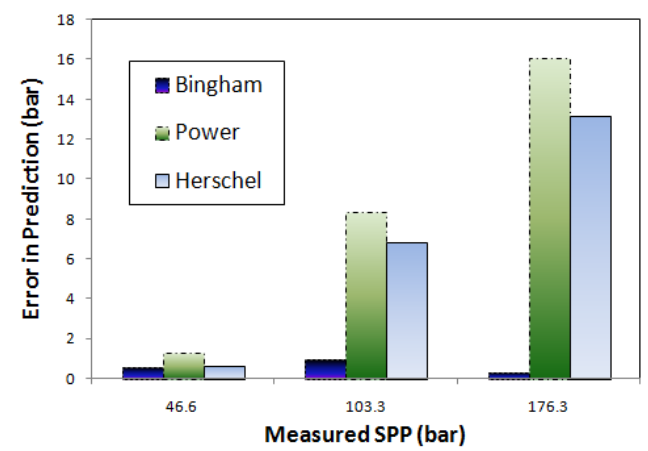

Figure 3: Absolute error in predicted SPP by the three NonNewtonian models

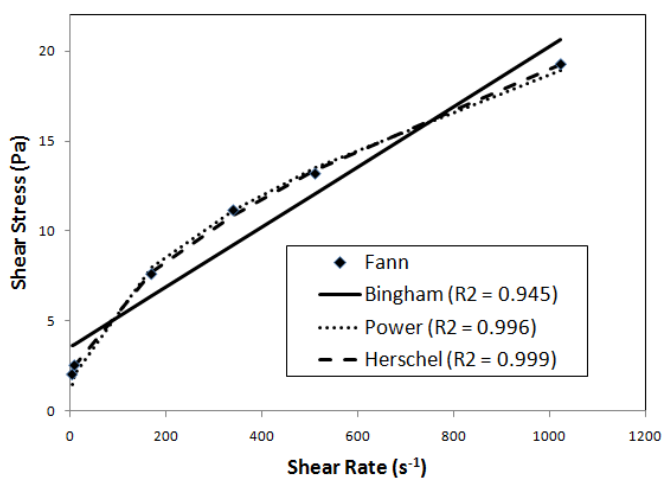

Figure 4: Rheogram for the non-Newtonian models

pressure drop relations used are based on a number of simplifying assumptions, such as concentric annular and circular sections, non-rotating drillstring, isothermal conditions in the bore hole and steady state axial flow. These simplifying assumptions are not completely valid in real life [4]. The effect of pipe eccentricity, pipe rotation, and temperature and pressure variations can have significant effect on frictional pressure drop in the annulus. The computational complexity in determining the effects of these parameters on SPP may not be justified sometime for simple vertical wells drilled onshore. However, their effects are significant while drilling deviated holes in complex formations where the mud window is narrow. They also need to be taken into consideration for High Pressure High Temperature (HPHT) wells where high temperature conditions cause the fluid in the wellbore to expand and high pressure conditions cause fluid compression [14]. These limitations of the conventional approach have led to the development of alternative methods for SPP prediction. One such method known as instance-based reasoning, a machine learning method, is discussed by Chowdhury [15].

\section{Conclusion and Recommendations}

Four rheological models are tested to find out their ability of predicting SPP with sufficient accuracy using the conventional one dimensional frictional pressure drop relations. The Bingham plastic model is found to produce SPP estimates considerably close to the measured values for the drilling data considered. However, it should be kept in mind that the conventional approach of SPP prediction is based on a number of simplifying assumptions which are not completely valid in real life.

There is scope for further work. The conventional approach of SPP prediction can be tested with Fann viscometer data collected using different types of mud samples, borehole geometries and surface equipment dimensions.

\section{References}

[1] Okafor M and Evers J, Experimental Comparison of Rheology Models for Drilling Fluids, in SPE Western Regional Meeting, 1992

[2] Maglione R and Robotti G, Field Rheological Parameters Improve Stand Pipe Pressure Prediction While Drilling, in SPE Latin America/Caribbean Petroleum Engineering Conference, 1996

[3] De Sa C, Martins A and Amaral M, A Computer Programme for Drilling Hydraulics Optimisation Considering Realistic Rheological Models, in European Petroleum Computer Conference, 1994

[4] Bourgoyne Jr A, Millheim K, Chenevert M and Young Jr F, Applied Drilling Engineering, SPE Textbook Series Vol. 2, SPE, Richardson, TX, 2003

[5] Rabia H, Oilwell drilling engineering: principles and practice, Graham \& Trotman, London, 1985

[6] Aadnøy B and Ravnøy J, Improved pressure drop/flow rate equation for non-Newtonian fluids in laminar flow, Journal of petroleum science \& engineering, 1994. 11(3):pp. 261-266

[7] Islam A, Drilling Fluid Rheology Note, Lecture Note, IPT, NTNU, Trondheim, 2008

[8] Chowdhury D, Hydraulic Pressure Losses in Drilling Fluid, MSc Project Report (for the course TPG 4520), IPT, NTNU, Trondheim, 2008

[9] Skalle P, Compendium: Drilling Fluids and Borehole Hydraulics, Tapir Akademisk Forlag, Trondheim, 2005

[10] Graves W and Collins R, A New Rheological Model for NonNewtonian Fluids, Paper SPE, 1978. 7654

[11] Whittaker A, Theory and application of drilling fluid hydraulics, Reidel, Boston, 1985

[12] Modi PN and Seth SM, Hydraulics and Fluid Mechanics Including Hydraulic Machines, Standard Book House, New Delhi, 14th edition, 2002

[13] Chilingarian GV and Vorabutr P, Drilling and Drilling Fluids, Elsevier, Amsterdam, updated text book edition, 1983

[14] Harris O and Osisanya S, Evaluation of Equivalent Circulating Density of Drilling Fluids Under High Pressure/High Temperature Conditions, in SPE Annual Technical Conference and Exhibition, 2005

[15] Chowdhury D, Prediction of Standpipe Pressure Using Real Time Data, MSc Thesis Report (for the course TPG 4920), IPT, NTNU, Trondheim, 2009 


\section{Appendix}

Table 1: Casing and hole dimension

\begin{tabular}{cc|cc|c}
\hline \multicolumn{2}{c|}{$\begin{array}{c}\text { Casing Size } \\
\text { (in) }\end{array}$} & \multicolumn{2}{|c|}{$\begin{array}{c}\text { Depth } \\
\text { (m) }\end{array}$} & $\begin{array}{c}\text { Open Hole } \\
\text { (in) }\end{array}$ \\
\hline OD & ID & From & To & \\
\hline 20 & 19 & 0 & 598 & \\
& & 598 & 798 & $17^{1} / 2$ \\
\hline
\end{tabular}

Table2: Mud rheology measurements at standard conditions

\begin{tabular}{|c|c|c|c|}
\hline $\begin{array}{l}\text { Rotor Speed } \\
\quad(\mathrm{rpm})\end{array}$ & \multicolumn{3}{|c|}{$\begin{array}{c}\text { Fann Dial Reading } \\
\text { (degree) }\end{array}$} \\
\hline 600 & \multicolumn{3}{|c|}{38} \\
\hline 300 & \multicolumn{3}{|c|}{26} \\
\hline 200 & \multicolumn{3}{|c|}{22} \\
\hline 100 & \multicolumn{3}{|c|}{15} \\
\hline 6 & \multicolumn{3}{|c|}{5} \\
\hline 3 & \multicolumn{3}{|c|}{4.5} \\
\hline \multicolumn{4}{|c|}{ Table 3: Drillstring dimension } \\
\hline \multirow[t]{2}{*}{ Type } & \multirow{2}{*}{$\begin{array}{l}\text { Length } \\
(\mathrm{m})\end{array}$} & \multirow{2}{*}{$\begin{array}{l}\text { OD } \\
\text { (in) }\end{array}$} & \multirow{2}{*}{$\begin{array}{l}\text { ID } \\
\text { (in) }\end{array}$} \\
\hline & & & \\
\hline Stand Pipe & 20 & - & 4 \\
\hline Rotary Hose & 20 & - & 3.5 \\
\hline Swivel & 3.5 & - & 3.5 \\
\hline Kelly & 12 & - & 3.5 \\
\hline Drill Pipe & 513.6 & 5 & 4.28 \\
\hline Heavy Wate & 137 & 5 & 3 \\
\hline Drill Collar & 70 & 9 & 3 \\
\hline Drill Collar & 77 & $11^{1 / 4}$ & 3 \\
\hline Bit & 0.4 & Nozzle & \\
\hline & & $3 \times 15$ & \\
\hline & & $1 \times 14$ & \\
\hline
\end{tabular}

Table 4: Measured SPP for

\begin{tabular}{cc}
\multicolumn{2}{c}{ different flow rates } \\
\hline $\begin{array}{cc}\text { Pump Rate } \\
\text { (liter/min) }\end{array}$ & $\begin{array}{c}\text { SPP } \\
\text { (bar) }\end{array}$ \\
\hline 1640 & 46.6 \\
2460 & 103.3 \\
3270 & 176.3 \\
\hline
\end{tabular}

Table 5: Rheological constants

\begin{tabular}{crcc}
\hline Newton & Bingham plastic & Power law & Herschel-Bulkley \\
& & & \\
\hline$\mu=0.022$ Pas & $\tau_{y}=3.57 \mathrm{~Pa}$ & $\mathrm{k}=0.671$ & $\tau_{y}=1.15 \mathrm{~Pa}$ \\
& $\mu_{p}=0.0167 \mathrm{Pas}$ & $\mathrm{m}=0.482$ & $\mathrm{k}=0.362$ \\
& & $\mathrm{~m}=0.565$ \\
\hline
\end{tabular}

Table 6: Predicted SPP

\begin{tabular}{|c|c|c|c|c|c|}
\hline Flow rate (liter/min) & Measured SPP (bar) & \multicolumn{4}{|c|}{ Predicted SPP (bar) } \\
\hline 1640 & 46.6 & 493.24 & 47.14 & 45.32 & 45.95 \\
\hline 3270 & 176.3 & 1721.75 & 176.6 & 160.25 & 163.15 \\
\hline
\end{tabular}

